\title{
The role of template matching in the attribution of immorality
}

\author{
Nicolas Restrepo
}

07 June, 2021

\begin{abstract}
The sociology of culture provides tools to weigh in on key interdisciplinary debates that hinge around categorization and its underlying processes. For example, at present, there is widespread debate about how individuals come to perceive events as immoral. In this paper, I use sociological approaches to cultural meaning to test one of the leading theories of moral cognition: the idea that individuals attribute immorality through template matching. I use spatial measures of cultural meaning to define and locate a prototypical moral wrong. I then test the theory of template matching, and find evidence that distance from the typical moral transgression - in semantic space - is related to the time it takes to categorize an event as immoral or harmful. I then test these results on a corpus of naturally occurring text to assess their external validity. These studies provide empirical evidence supporting the notion that the attribution of immorality occurs through template matching. Furthermore, they also serve to illustrate that productive conversations can emerge when we take the insights that sociologists of culture and cognition have reached in the past few decades out of our disciplinary boundaries.
\end{abstract}




\section{Introduction}

The sociology of culture and cognition has often been at its most insightful when examining categorization. The field has made important contributions to our understanding of how symbolic boundaries are traced between groups of individuals ((Brubaker, Loveman, and Stamatov 2004), (Lamont 1992)), how social categories emerge around certain prototypes (Martin and Desmond 2010), and how seemingly disparate elements are brought together under the same classificatory scheme (Zerubavel 1996). There have been recent calls for cultural sociologists to get more involved in interdisciplinary dialogue (Vaisey and Valentino 2018). In this paper, I argue that sociological work around cultural categories - and their measurement - provides an opportunity for such interventions. I demonstrate this by using sociological approaches to measuring cultural meanings to test one of the leading theories of how individuals classify events as immoral.

There is widespread debate about how actors identify and categorize moral transgressions. At present, one of the most plausible explanations is that we attribute moral wrongdoing through template matching (Schein and Gray 2018). In other words, when we perceive an event, we compare it to our idea of what the typical moral transgression looks like. If the perceived event closely matches our cognitive template of an immoral act, then we are likely to view it as a transgression. Even though this theory has gained traction, the evidence to support it remains limited.

One of the main obstacles for testing the role of template matching in moral decision-making is that operationalizing the notion of a "typical" moral transgression is a difficult problem. I contend we can overcome this issue if we approach it through a sociological lens, acknowledging that mental templates are cultural objects that are - to a higher or lesser extent - shared among members of the same group. Just as cultural schemas surrounding poverty affect our evaluations about who is worthy of financial aid (M. F. Hunzaker and Valentino 2019), the meanings about who represents a vulnerable victim or what constitutes a harmful action 
inform our moral judgments (Bergstrand 2019). The fact that cultural meanings surrounding moral transgressions are shared means we can measure them; by accessing these collective understandings, it is possible to provide a principled definition of a "typical" moral wrong.

In this paper, I take advantage of the fact that the bulk of research on moral cognition has used hypothetical vignettes. Using Osgood et al.'s (1975) approach to measuring cultural meaning, I produce "translations" of these vignettes into comparable units and examine their connotative structures. This accomplishes two goals: first, I identify shared semantic features among the scenarios in order to come up with a principled way of outlining what a prototypical immoral act looks like; second, I calculate the extent to which the vignettes match this template, aiming to shed light on how distance from the exemplar is related to the attribution of immorality. I examine the informativeness of this measure of distance in survey data but also in naturally occurring text, showing its considerable explanatory power. By using tools that have become increasingly widespread among sociologists interested in culture, then, it is possible to provide an empirical test of one of the leading theories of moral cognition.

My argument will have the following structure. First, I outline a central debate that currently exists in interdisciplinary work about moral cognition. Second, I explain how we can use sociological approaches to cultural meaning to adjudicate this debate, by translating vignettes into a format amenable for the analysis of their underlying semantic features. Third, I describe the methods and results of Study 1, which shows that the translations of the scenarios are interpreted consistently by respondents. Fourth, I discuss the design and findings of Study 2, where I use reaction time data to examine see if the pattern of results is consistent with template matching. Fifth, I assess the external validity of the results from Study $1 \& 2$ by testing them in a large corpus of naturally occurring text. Lastly, I close by discussing the implications and limitations of the analysis, as well as the potential avenues for research that it opens up. 


\section{Theoretical background}

\section{Discrete categories vs. Underlying continuums}

Although research on how individuals come to perceive actions as immoral has been disjointed, scholars have reached some common ground. Evidence from different fields suggests that actors do not reach moral judgments through logical deliberations, weighing their commitments to universal principles against the immediate implications of their actions (Greene 2014, 2015). Rather, they rely on "gut feelings" to instinctively decide what is right or wrong. We intuitively know what is impermissible, even if we are not capable of articulating why (Haidt and Joseph 2004). Questions remain, though, about the underlying cognitive processes that make individuals such swift arbiters of immorality, and about how these vary across social contexts. In answering these questions, scholars have generally put forward two types of explanations: one claims that there are qualitatively different types of moral transgressions, and that actors use distinct cognitive strategies to understand them; another suggests that moral wrongs are all judged based on one underlying logic.

Explanations that contend that morality consists of differentiated domains have emerged as a response to an empirical puzzle: the fact that there are events that individuals find immoral, but which do not involve harm (Haidt, Koller, and Dias 1993). Dietary and sexual taboos are examples of these seemingly harmless wrongs. The most prominent theory in this line of work is Moral Foundations Theory (henceforth MFT) (Graham et al. 2013). MFT scholars argue that the diversity of moral judgments is explained by the fact that morality is underpinned by five distinct foundations: authority, fairness, loyalty, purity, and harm. We attribute immorality differently depending on the moral foundation that appears to be breached. For instance, seeing a soccer player bend the rules to their favor is substantively different that watching them deliberately try to injure a rival. Both are immoral acts but while the former evokes normative sentiments about fairness, the latter is concerning because of the harm that might be inflicted on the other player. 
A more unified conceptualization of morality - the one that MFT precisely argues against has regained traction throughout the last decade. Evidence that shows that the five moral foundations are all highly correlated has been at the center of this theoretical push (Gray and Keeney 2015b). This suggests that transgressions are cut from the same cloth and cannot be parceled out into different groups. Gray and Schein (2018) contend that the unifying dimension of moral decision-making is harm. Their work shows that when individuals are asked to envision immoral acts, they tend to bring up quintessentially harmful practices, and that when asked to explain what makes certain actions immoral, they tend to resort to ideas of harmfulness (Gray and Keeney 2015a; Schein and Gray 2018). Individuals, thus, judge actions as immoral insofar as they can perceive them as harmful. Perceptions of harm even mediate our understanding of practices where no entities appear to be directly affected: incestual marriages, for instance, are often described as harming the health - the gene pool of the group (Schein and Gray 2018). Their argument is that, regardless of the superficial differences between moral violations, the central mechanism through which individuals come to see them as immoral is harm.

\section{Template matching and moral cognition}

There is a working theory of moral cognition that is congruent with a more unified vision of morality and it relies on the notion of template matching (Gray, Young, and Waytz 2012). Template matching is a cognitive process whereby individuals categorize information by contrasting it with salient mental exemplars built from experience and socialization (Schein and Gray 2018; Harnad 2017). Thus, category membership is not ascribed through inclusionary/exclusionary criteria but rather through proximity to a mental template. For example, when individuals are asked to picture a bird, they are more likely to envision something that looks like a robin than an ostrich (Rosch 1988). What the proponents of this position argue, then, is that when we are asked to picture a moral transgression, we have a particular mental image of what that looks like (Schein and Gray 2018). When 
confronted with an event, we compare it with this cognitive template in order to assess its harmfulness and immorality. Moral cognition, then, does not entail sorting an event into discrete categories. Instead, it involves placing a violation on a continuum, more or less distant from our mental template of a typical moral wrong.

Proponents of this theory provide a description of this mental template, but details remain opaque and the empirical evidence limited. Immoral actions, they argue, have a dyadic structure: they involve an intentional agent directing a damaging behavior towards a vulnerable patient (Schein and Gray 2018). An event that would resemble such a template would be a calculating criminal murdering a child. When we witness an event, we compare it to this template and assess its immorality accordingly. Though plausible, this definition remains vague. It is unclear how we can define what constitutes a vulnerable victim or a damaging behavior without relying on tautologies. Therefore, we do not know how to gauge whether an event fits into this template, let alone how to calculate how practices might deviate from it. Furthermore, we lack direct empirical tests of whether and how template matching mediates the attribution of immorality. Here, I show that cultural sociology helps address this gap; its tools allow us to empirically test this process by measuring the cultural meanings of moral transgressions.

\section{The social meanings of moral transgressions}

The central challenge in the effort to test this theory is outlining a principled definition of the prototypical moral violation. An important step towards that goal is to realize that mental templates are eminently cultural (M. B. F. Hunzaker 2016; M. F. Hunzaker and Valentino 2019). The cultural landscapes in which we are embedded shape how we construct certain categories, and the exemplars around which they are organized (Zerubavel 1996). This is known territory for sociologists: there is extensive work about how symbolic boundaries are traced and how classificatory systems emerge. This extends to views of categorization that rely on template-matching: recent research suggests that our ideas of what the typical African 
American (Monk 2019) or benefit claimant looks like (M. F. Hunzaker and Valentino 2019; Martin and Desmond 2010) vary depending on the social positions we occupy. By virtue of inhabiting the same social environments, then, members of a group acquire relatively similar templates.

The key implication here is that the fact that mental templates are shared cultural objects makes them measurable. We can treat the effort to outline a template of moral transgressions in the same way as scholars have attempted to retrieve schematic associations (M. F. Hunzaker and Valentino 2019) or prototypes in other social categories (Schröder et al. 2013). In fact, recent work by Bergstrand (2019) suggests that this effort is worthwhile, showing that there are patterns in how individuals judge who is a more typical victim and thus more worthy of help. Following this line of work, we can examine the underlying cultural meanings associated with immoral acts in order to look for regularities, piecing together an outline of a prototypical moral transgression. There are two main challenges in this approach: first, we are dealing not with a single entity but with a dyadic structure, consisting of an agent directing a behavior towards a recipient; second, we need to find rigorous measures of cultural meaning that we can map onto these different components. Given fortuitous theoretical overlaps, it is possible to use the dictionaries of affective meanings collected by Affect Control Theory (henceforth $\mathrm{ACT}$ ) scholars to address these challenges.

ACT posits that individuals act in order to maintain the cultural meanings associated with their identities (David R. Heise 2007). Due to the central role that meaning plays in this theory, ACT scholars have developed rigorous strategies to measure it. For the purposes of this paper, it is worth highlighting three ideas that capture ACT's approach to meaning: first, that identities and behaviors have distinct cultural meanings; second, that these meanings are widely shared among members of the same group; and third, that these semantic structures can be reduced to a set of measurable dimensions (David R. Heise 2007; M. B. F. Hunzaker 2016). In order to operationalize the latter, this theory incorporates Osgood et al.'s (1975) 
work, arguing that the meaning of any concept can be effectively distilled to three dimensions: evaluation, potency, and activity. In other words, we need three pieces of information to broadly capture the cultural meaning of a concept: we need to know whether it is good or bad (evaluation), whether it is weak or strong (potency), and whether it is lively or quiet (activity) (Charles E. Osgood 1969). Extensive cross-cultural work has shown that these three dimensions broadly capture connotative meanings, both across cultures and throughout time (Charles Egerton Osgood et al. 1975).

Using these dimensions, it is possible to collect rigorous measures of the meanings that groups attach to concepts. These aggregate meanings are collected by asking respondents to rate concepts on three, nine-point scales corresponding to each of the dimensions (David R. Heise 2014). These measures are then aggregated, and the average is computed, yielding the evaluation, potency, and activity (EPA) values for each concept. These EPA profiles are accurate and informative. Babies, for instance, are very good, very weak, and somewhat lively, while murderers are depicted as very bad, very powerful, and slightly lively. This framework, then, helps render cultural meanings measurable and formalizable. As part of their research agenda, ACT researchers have collected large dictionaries of affective meanings. I use the online ACT dictionary collected in 2015 (Robinson et al. 2016) to map reliable measures of cultural meaning onto the different components of transgressions.

Research on moral cognition has primarily used fictional scenarios to examine how actors assess immorality. In these studies, respondents are presented with a series of hypothetical events and they must decide how immoral or harmful they are. These vignettes tend to share a dyadic grammatical structure much like the one discussed above: they often involve an actor directing a behavior towards a recipient. This structure (actor-behavior-object) is also the main unit of analysis of ACT. This means that the dictionaries of affective meanings contain many of the terms we would need to translate widely used fictional scenarios into a format that allows for the analysis of their connotative meanings. Let me provide an example. 
A scenario that has been shown (Clifford et al. 2015) to be interpreted reliably is:

You see a teacher hitting a student's hand with a ruler for falling asleep in class.

Ascribing appropriate EPA profiles to each component of the sentence, we can produce the following translation:

$$
\begin{aligned}
& \text { Teacher }[\mathrm{E}=2.5 ; \mathrm{P}=2.31 ; \mathrm{A}=0.32] \text { hits }[\mathrm{E}=-2.66 ; \mathrm{P}=1.30 ; \mathrm{A}=2.12] \text { lazy } \\
& \text { student }[\mathrm{E}=-0.42 ; \mathrm{P}=-0.76 ; \mathrm{A}=-1.56]
\end{aligned}
$$

This format provides a wealth of additional information about these vignettes. Much like an $\mathrm{x}$-ray, the translation allows to go below the level of denotative meanings and to examine the underlying cultural structure of the event. This information, in turn, is useful in the effort to understand template matching in the context of moral cognition. For instance, it is possible to examine what semantic features play an important role in the attribution of immorality. Furthermore, we can look for identifiable attributes of typically harmful actions such as murder - and prototypically vulnerable victims - such as children (Bergstrand 2019). Thus, by going down to the level of connotative meanings, we gain valuable information that will prove essential for understanding the relationship between moral cognition and template matching.

In the following studies, I use this approach to assess the prototypicality of moral transgressions and to analyze how deviations from those regularities shape moral decision-making. In Study 1, I show that the "translated vignettes" are interpreted reliably, and I analyze which semantic attributes - e.g. the agent's evaluation or the object's activity - are most informative for understanding the attribution of immorality. This, in turn, helps me reach a principled formalization of what the template of an immoral act looks like. In Study 2, I examine if an event's distance from this template is predictive of how long it takes individuals to categorize it as immoral or harmful. Using reaction time data, I test whether the attribution of immorality occurs through template matching. In Study 3, I derive a hypothesis from the previous analyses and use natural language processing tools to test it in a large corpus 
of text, comprising all Vox articles published before March 21st of 2017 (the data-set was compiled by Elena Zheleva, and it's available here). This allows me to assess the external validity of the results obtained in the first two studies.

\section{Study 1}

\section{Data and Methods}

I collected the data for this study through the Prolific platform. Prolific is a crowdsourcing website that allows researchers to connect with participants interested in completing different types of tasks. This platform has advantages over its counterparts: it focuses on data quality and has a replenishing pool of participants (Peer et al. 2017). The latter is important because it ensures that respondents are not so familiar with surveys and experiments that completing such tasks becomes a purely mechanistic endeavor. In total, I reached out to 205 participants and, after removing cases with missed attention checks, the total number of usable responses was 194.

For this study, I translated 25 vignettes into the format that allows me to explore their connotative meanings. In order to compile this list, I drew on the work of Gray and Keeney (2015a), Young and Saxe (2011), and Clifford et. al. (2015). I chose scenarios that had been previously used and that I could translate using ACT's dictionaries of meaning. I also decided to include five scenarios for each of the five moral foundations. Although the idea that moral wrongs belong to distinct categories has been recently criticized, it remains useful to examine whether systematic differences exist. The full list of translations is provided in the supplemental materials. I presented each participant with the 25 translated scenarios and asked them to rate how immoral, harmful, and unexpected they were. They provided their ratings using five-point Likert scales that went from, for example, "not harmful at all" to "extremely harmful." The decision to include these three scales was based on previous work, which had examined these dimensions (Gray and Keeney (2015a); Schein and Gray (2018)). 
This study has two main goals: first, to confirm that the translations are interpreted appropriately and that they produce results that are consistent with previous findings; second, to examine which semantic features have the biggest impact in perceptions of immorality. In order to accomplish the former, I compare the average immorality rating of each scenario with its harmfulness and unexpectedness ratings. Previous work suggests that both relationships should be positive. To address the second goal, I fit cross-classified, multi-level models predicting immorality ratings on the event's semantic components - like actor's evaluation or object's potency. These models include varying intercepts for individuals and scenarios in order to account for systematic differences between events and respondents. The results will give me a principled basis to reach a formal definition of a prototypical moral transgression.

\section{Results}

Figure 1 shows the relationship between the average immorality of each scenario and its average harmfulness and average unexpectedness. The results further reaffirm the close relationship between immorality and harm. Moreover, the positive relationship between immorality and unexpectedness resonates with Gray and Keeney's (2015a) idea that severe transgressions tend to be perceived as more unexpected because they often entail the breaching of social mores. For the purposes of this study, the most important point is that the translated scenarios are interpreted consistently. There are encouraging patterns that are consistent with findings in the literature: the "harm" transgressions tend to be rated as the most severe, while the "purity" violations rank among the most unexpected. This suggests that there was not a significant loss of information when the vignettes were translated into the new format. 
Figure 1

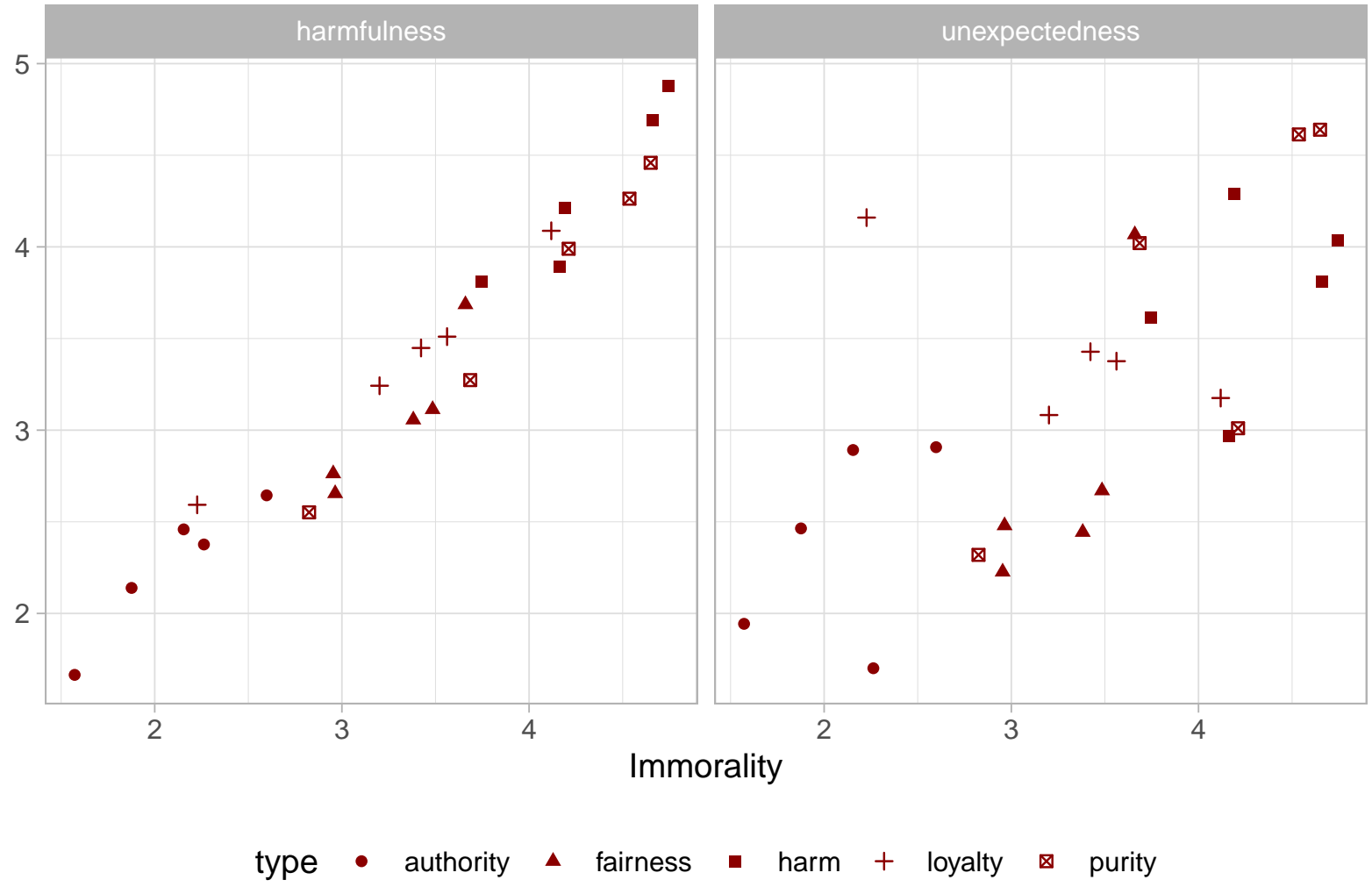

To assess the relationship between semantic features and perceptions of immorality, I regress the latter on the EPA values of each element of the actor-behavior-object triplet. I fit various model specifications - e.g. accounting for the respondents' political identification and then select the most informative one using LOO. LOO is a widely accepted measure of out-of-sample deviance that indicates how predictive a model would be in a new dataset; the results are available in the supplemental materials.

The most informative model is a cross-classified model that includes all semantic features but that forgoes additional covariates, such as the gender or political identification. Figure 2 shows the coefficient plots for two models. In the second model, I exclude the so-called "purity" scenarios from the analysis, following previous literature that has argued that these transgressions are distinctive, either because they a fundamentally different type of violation or simply because they are unconventional. Both models indicate that there are three semantic features that are particularly informative when considering the immorality of an event: the 
behavior's evaluation, the behavior's potency, and the object's potency. Though there is uncertainty around some of these coefficients - especially in the first model - both analyses point at the informativeness of the same three semantic components. Reassuringly, these results are consistent with Gray and Schein's (2018). Negative and potent behaviors are perceived as more immoral. In turn, as the object's potency increases, the immorality of an acts tends to decrease. This offers a more concrete definition of the notion of a "vulnerable patient." As the recipient of an action appears less potent, said action is considered more immoral.

Figure 2

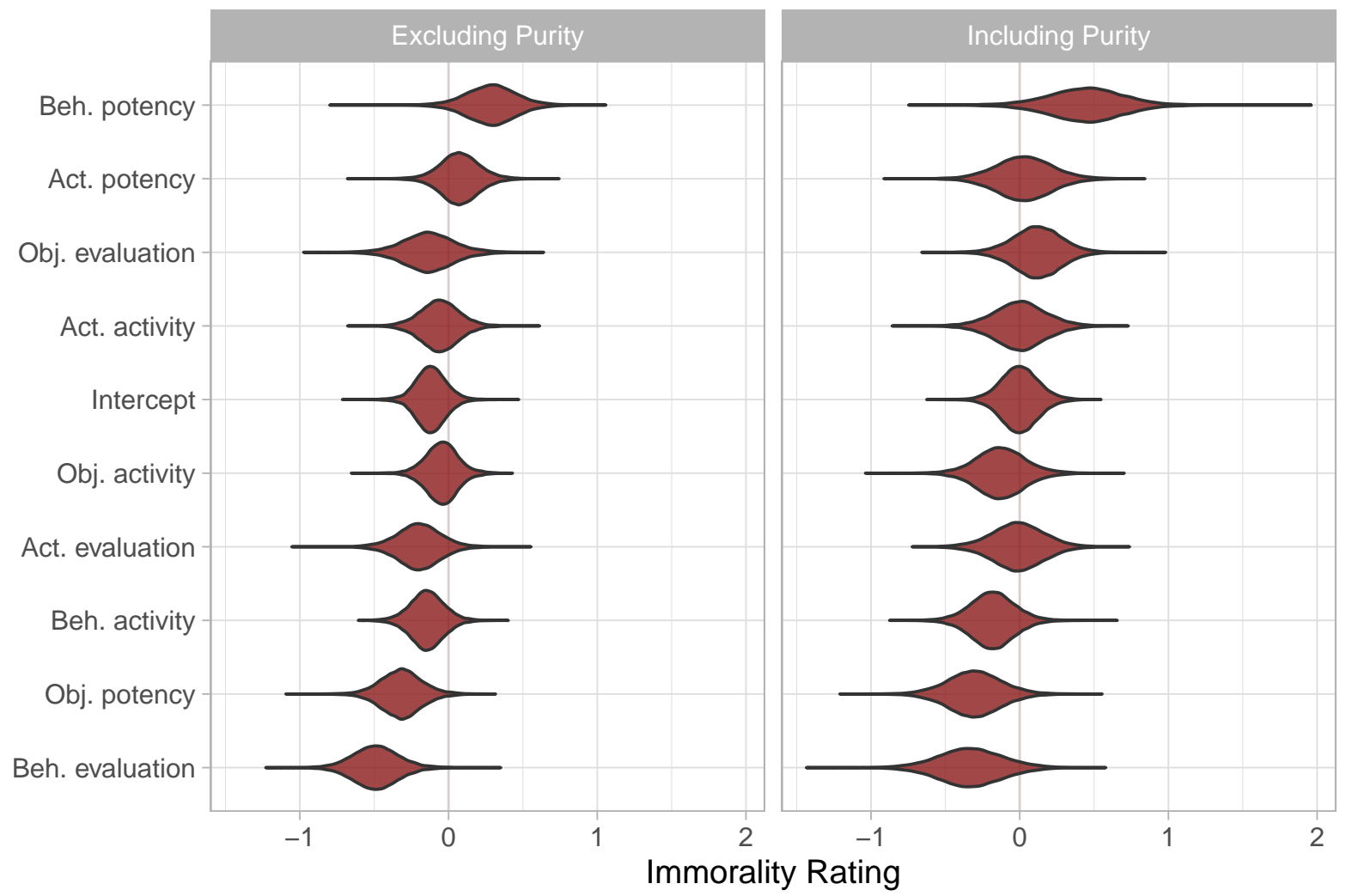

Establishing the importance of these three features allows me to place events in a threedimensional space in order to compare them. Figure 3 shows this space. This is key for two main reasons. First, it helps me reach a principled definition of a prototypical moral transgression: it should involve a potent and negative event directed at a very weak object. Using the terms that I have tested, the most appropriate event I can formulate is: 
A person kills a child.

The second reason why building such a three-dimensional space is important is because it allows me to calculate distance between the scenarios and the template defined above. This is the key piece of information that helps me test whether template matching mediates the attribution of immorality and/or harmfulness.

Figure 3

$$
\diamond \text { harm } \quad \nabla \text { purity } \otimes \text { fairness } * \text { authority } \diamond \text { loyalty }
$$

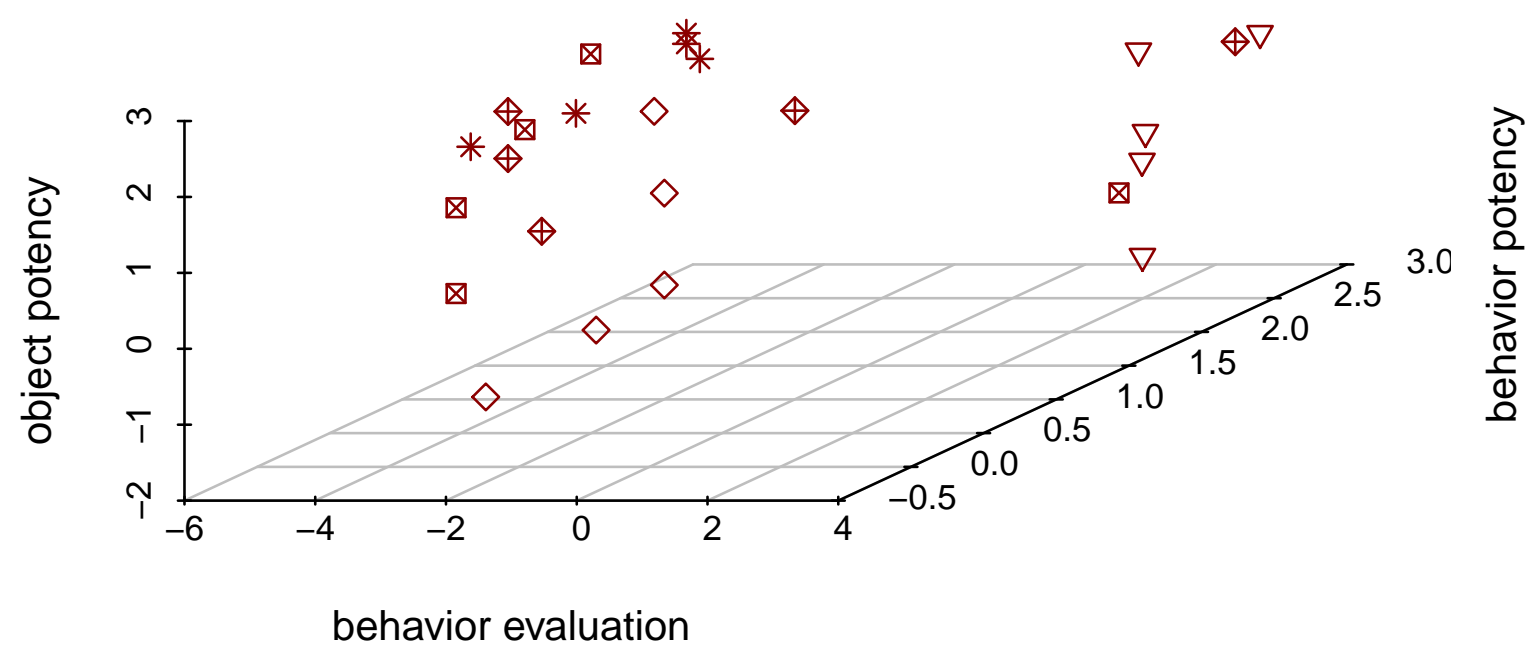

\section{Study 2}

\section{Data and Methods}

I also collected the data for this study through the Prolific platform. I reached out to a total of 200 participants and, after controlling for missed attention checks, there were a total 184 usable responses. In this study, I asked participants to categorize the same 25 scenarios as either immoral or not immoral, harmful or harmless. For each question, the scenario appeared in the middle of the screen and the dichotomous categories were shown at the bottom, on opposite sides of the page. Using their keyboards, the participants chose the category to which they think the event belongs. The outcome variable of interest is the time it takes 
respondents to categorize the violations. Reaction time here is meant to stand as a proxy for cognitive effort, with higher times reflecting that the categorization task is more difficult (Gray and Keeney 2015a; Moore 2017).

Each participant had to classify all scenarios on two dimensions and, therefore, I have a total of 9200 recorded times. To ensure that I could capture the initial reactions as reliably as possible, I designed the questionnaire so that participants had a ten-second limit to classify each scenario (with a one-second pause between questions). Out of all the responses, only 44 were above 10 seconds and 22 were below 1 second. Given that these data most likely reflect misclicks or technical difficulties, I deleted these cases.

The main goal of this analysis is to examine the relationship between distance from the prototypical moral transgression - a person kills a child - and the time it takes individuals to categorize the scenarios. I define distance from the template as the Euclidean distance between that point and a particular event in the three-dimensional space presented in Figure 3. Thus, if the prototypical transgression is $\mathrm{p}$, then distance for scenario $\mathrm{i}$ will be defined as:

$$
D_{i}=\sqrt{\left(B P_{p}-B P_{i}\right)^{2}+\left(B E_{p}-B E_{i}\right)^{2}+\left(O P_{p}-O P_{i}\right)^{2}}
$$

Finding that this distance is informative of reaction time would constitute novel evidence supporting the idea that moral cognition is underpinned by template matching. In order to test this proposition, I fit cross-classified, multi-level models where the main independent variable is distance from the prototype and the outcome variable is reaction time. The models include varying intercepts for both respondents and scenarios. They also include controls for length and readability. Reaction time data can be fickle and the length and complexity of scenarios certainly play a role in how long it takes respondents to categorize them. The models, then, include a term for the number of characters in each event and another coefficient with their respective Flesch-Kincaid readability score - an established measure of the level of 
education necessary to understand a text.

\section{Results}

The most informative model, in this case, is the one that depicts a quadratic relationship between distance from the prototype and reaction time. Although the LOO values are close, this specification is narrowly better than other functional forms, like positing a linear or a logarithmic relationship between the variables. This relationship appears to follow similar patterns for the classification of harmfulness and immorality, but there is a lot of uncertainty around the coefficients for the model predicting the former (see Figure 4). This is certainly an important limitation but given the close interconnection between immorality and harm, the results still provide insight for understanding template matching in the context of moral cognition.

Figure 4

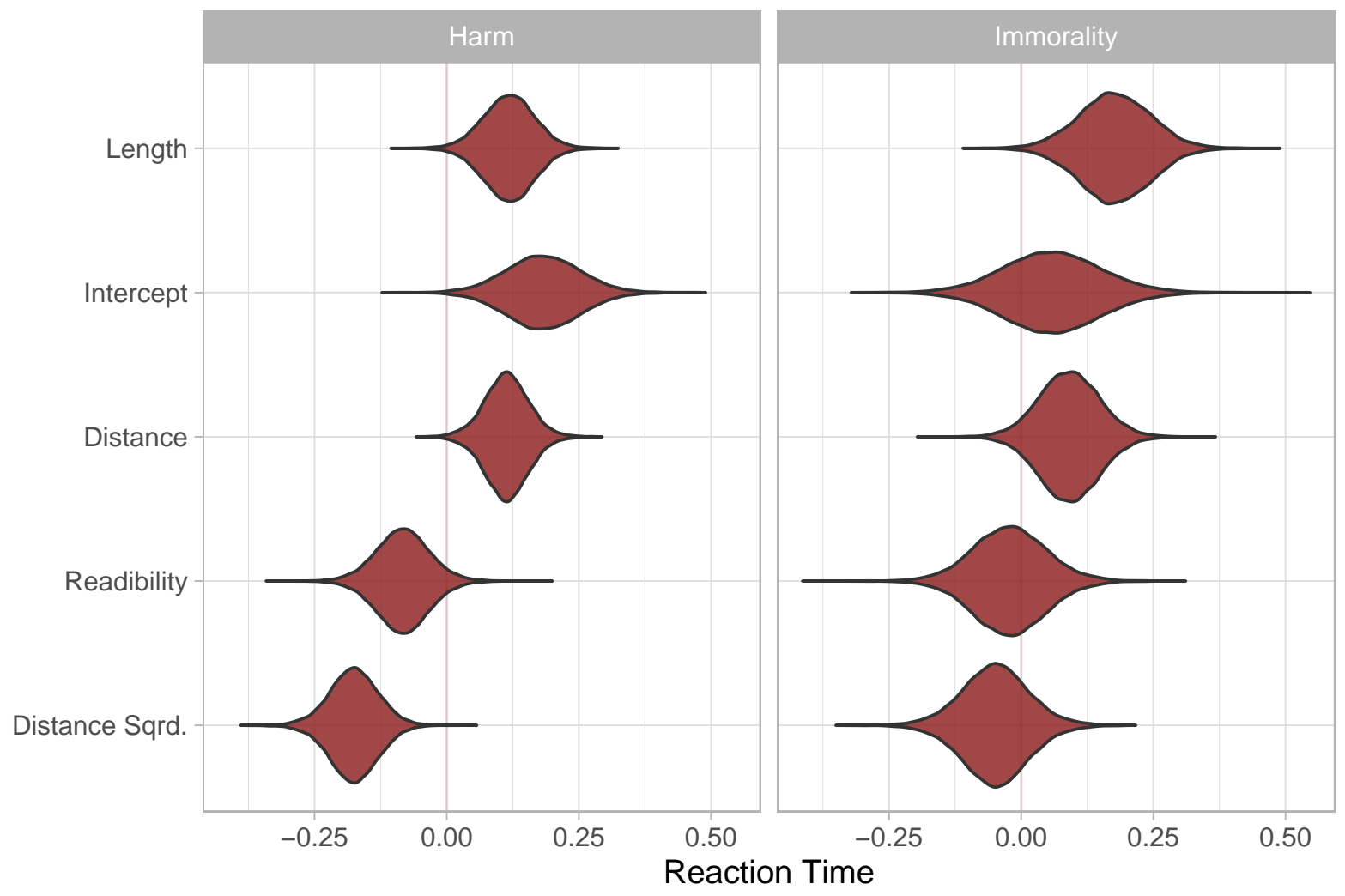

Figure 5 depicts the quadratic relationship: events that are very close and very far from the 
prototype take little time to classify. In other words, it is easy to see why prototypical events are harmful, while situations that are very far from that point - such as kissing one's spouse are quickly perceived as harmless. The results indicate that distance from a mental template might be informative for understanding moral cognition and that this relationship should be considered parabolically.

\section{Reaction Time by Distance from Prototype}

\section{Raw data and fitted model}

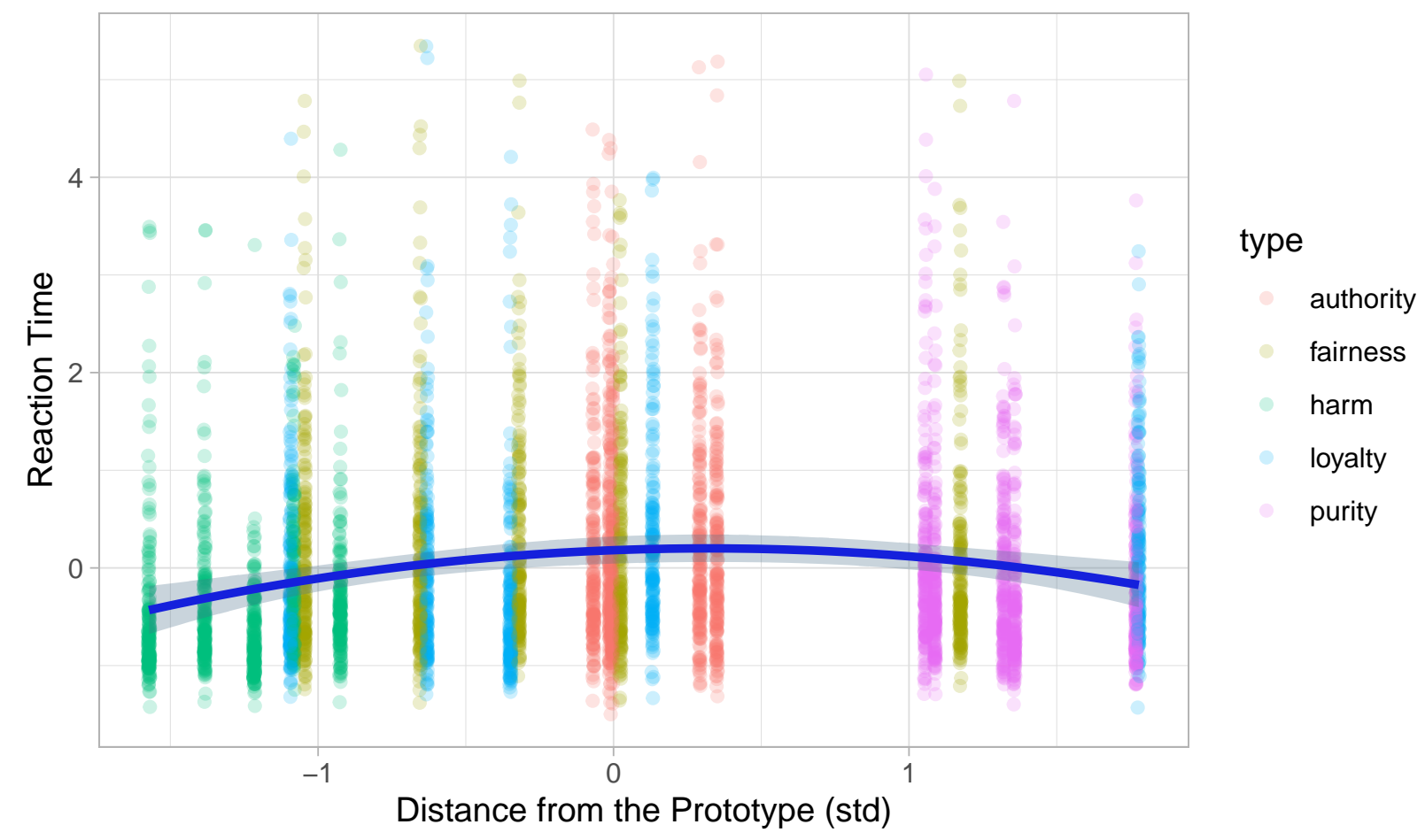

Figure 5

The model suggests that the scenarios that are most difficult to categorize are those that lie at a middling distance from the prototypical immoral act. This relationship between ambiguity and reaction time is also found in other parts of the data. For example, Figure 6 shows the predictions of a cross-classified, multi-level model that regresses reaction time of harmfulness on the proportion of participants who classified that event as harmful. I provide a full coefficient table in the supplemental materials, but the most important point is that this relationship is also parabolic: the events that had the most variance in how they were categorized took the longest to be classified. This finding furthers validates the decision to 
model the relationship between distance from the prototype and reaction as quadratic.

\section{Reaction Time by Proportion of Harmfulness}

\section{Raw data and fitted model}

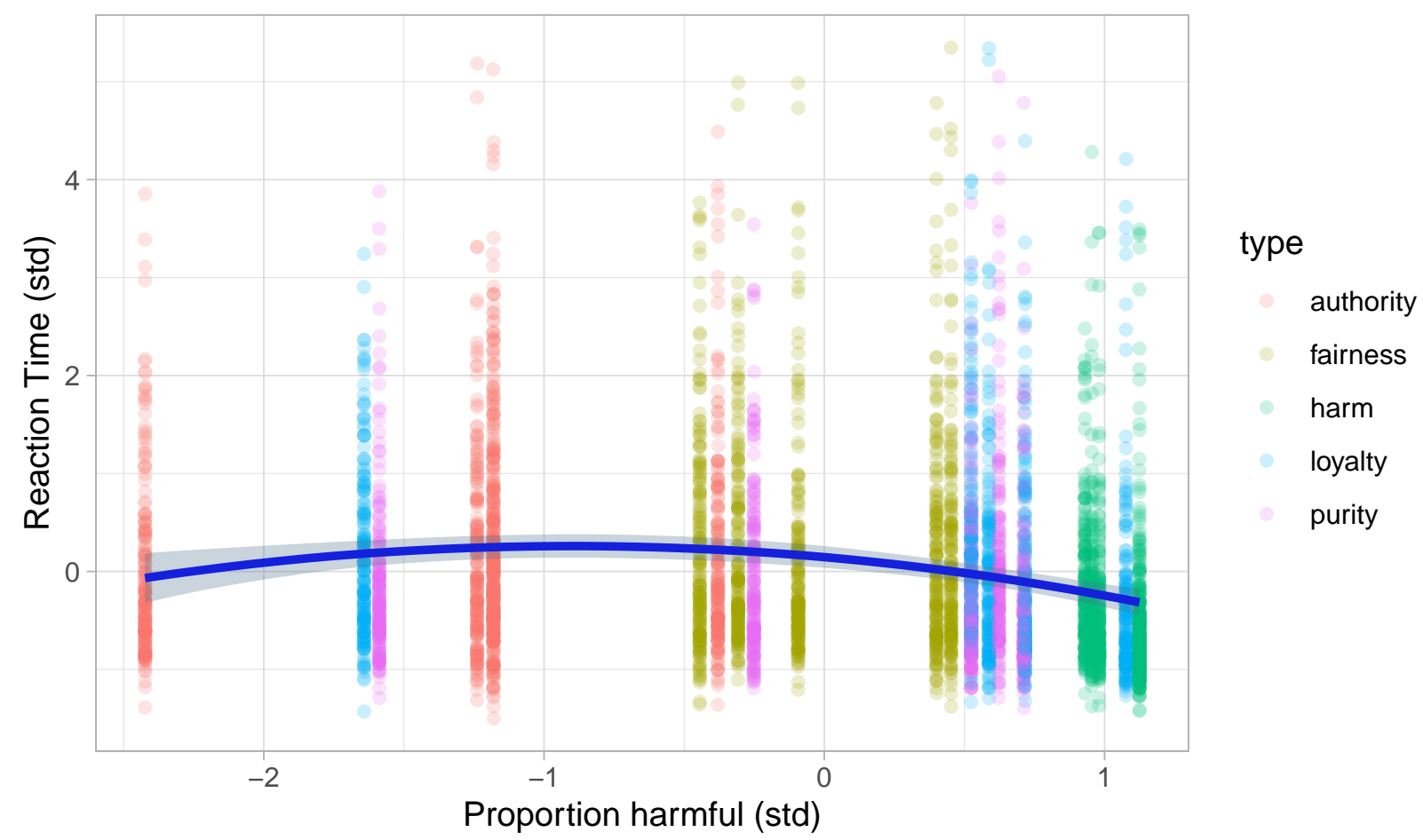

Figure 6

A concern with these results is that most of the scenarios that are very distant from the prototype are the so-called purity scenarios. Study 1 shows that these scenarios are consistently ranked among the most harmful. Thus, it is possible that these transgressions are driving the downward turn of the quadratic effect. To account for this possibility, I ran the same models taking these scenarios out of the sample and the quadratic relationship still held; these results are available in the supplemental materials.

More interestingly, however, it is possible to fit a logistic regression predicting whether an event was classified as harmful using distance from the prototype as the main independent variable. Now, instead of looking at how distance from the template is related to how long it takes to classify an event, I examine how that distance is associated with the actual harmfulness ratings. As Figure 7 shows, holding the length and readability of the vignettes constant, increases in distance from the prototype lead to a lower predicted probability of an 
event being classified as harmful. Again, it is important to re-emphasize that the so-called purity scenarios are both quite distant from the prototype and yet are very harmful. Thus, these scenarios are "out of place": their connotative structures do not seem to correspond to the harmfulness that participants attribute to them. As I will argue below, this fact carries important implications.

Is an event harmful?

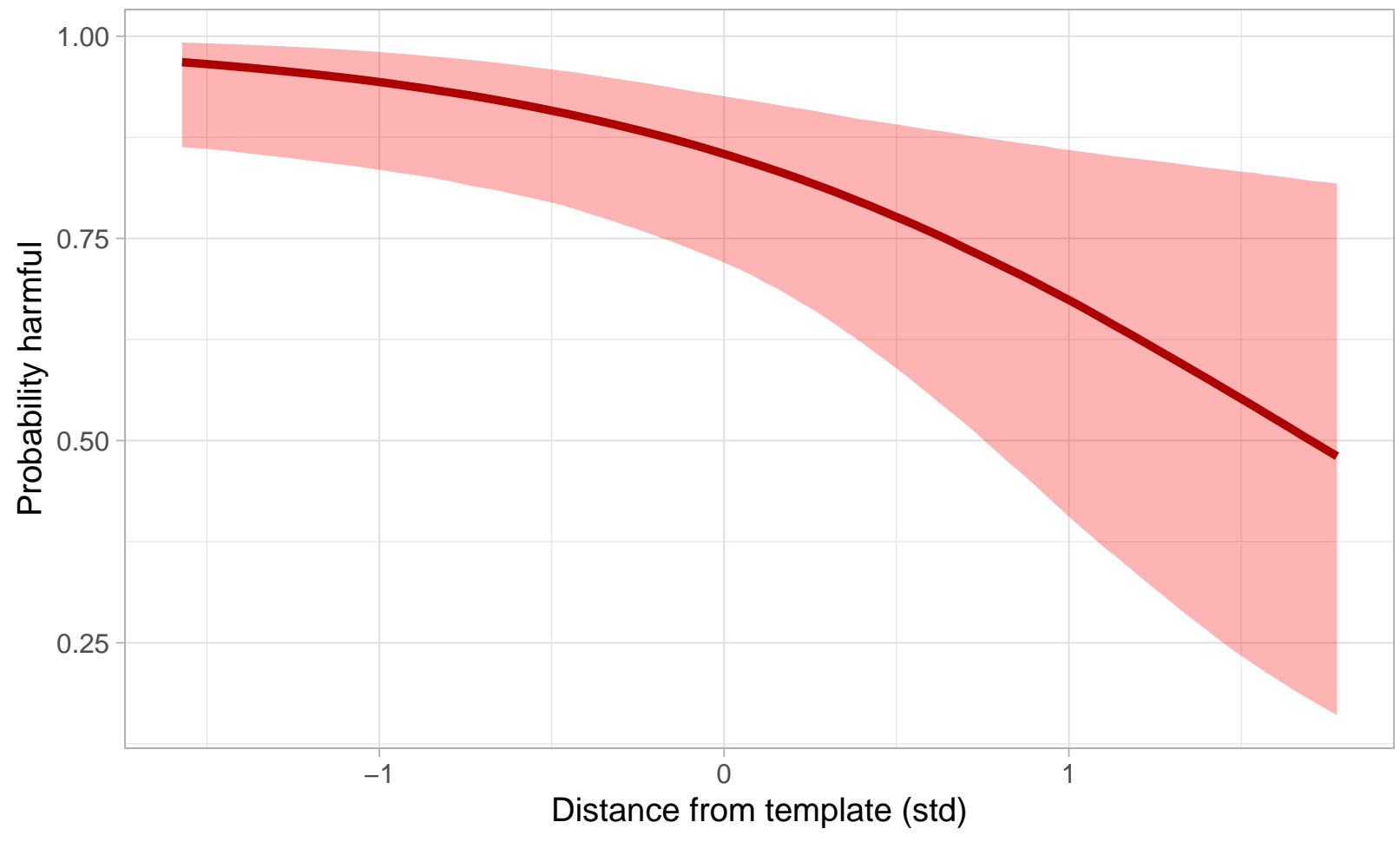

Shaded region is the $95 \%$ Confidence Interval

\section{Study 3}

While their results are insightful, the above studies share an important limitation: the events considered have really particular characteristics. Not only do they have similar semantic structures, but they were all curated to represent well-established moral violations. Study 1 and Study 2, then, seek to examine a general process of template matching using a homogeneous and idiosyncratic sliver of events. To rectify this, I examine whether distance from the template is a useful piece of information when analyzing a much wider set of events; 
a set that is not curated by researchers interested in moral violations, but rather extracted from naturally occurring text.

It is necessary, then, to translate the findings from Studies $1 \& 2$ into propositions that can be tested using natural language processing tools. Here, I choose to analyze whether distance from the moral template is informative of the kind of language that we expect to find around events in naturally occurring text. Using the results above as a starting point, it is possible to hypothesize that events that are close to the exemplar are more likely to be surrounded by words that would betray moral transgression. In other words, situations that involve an agent directing a negative behavior towards a vulnerable victim should co-occur with vocabulary related to immorality and harm. In the analysis below, I test the following hypothesis:

The rate of negative moral words around an event should be negatively related to its distance from the moral template.

Finding a relationship between how distant an event is from the exemplar and the amount of negative moral vocabulary around it would suggest that the former measurement has validity outside the confines of the surveys presented above. In the following section, I describe how I construct the data-set that I use to perform this analysis, as well as how I operationalize the notion of "negative moral words."

\section{Data and Methods}

For this analysis, I use a data-set consisting of all articles published by the news site Vox before the 21st of March 2017. This data-set was collected by Elena Zhelenko and made available to the public through the social platform data.world. In total, the corpus contains 23022 articles published between 2014 and 2017. These data prove particularly valuable for the problem at hand because Vox covers a wide array of topics, from economics to public policy and entertainment. This ensures that I am able to capture a relatively wide set of events, significantly broadening the scope of the studies above. 
In order to test the hypothesis, it is necessary to extract events from these articles; events that can be comparable in structure to the ones used in the previous studies. The automated identification of subject-verb-object triplets in naturally occurring text is an area of active research and the last few years have seen a proliferation of user-friendly and powerful tools designed for this task (cf. (Joseph et al. 2016)). In this project, I use the Python libraries spaCy and textacy, which offer automated parts-of-speech recognition tools that allow to identify and extract these triplets. As a concrete example, take the following sentence from one of the articles:

By the end of September 2013, the Obama administration had deported 1.83 million immigrants.

After extracting the subject-verb-object triplet, we are left with the following:

The Obama Administration had deported immigrants.

This sentence has a familiar structure: it consists of an agent directing a behavior towards an object. This common structure means that it is possible to map the EPA values collected by affect control theorists to the extracted triplets, provided that the definitions of their components are in the existent dictionaries. Thus, using tools for automated parts-of-speech recognition, it is possible to identify a set of the subject-verb-objects triplets that exist in the articles. These units, in turn, have a similar semantic structure to the events used in the studies above, providing a much broader and diverse testing ground to assess the external validity of the results.

After extracting all recognizable triplets from each article, I put the resulting data-set through several filters. I kept the triplets that had nouns as their objects and discarded the verbs that were preceded by a negation - e.g. "didn’t attack." I then filtered these triplets to identify the events for which there were existent EPA values. Here, it is important to recall that the measure of distance used in Study 2 takes into account the EPA scores of the behavior and the object. Therefore, these were the two components I took into account when mapping 
EPA scores to the identified triplets. The resulting data-set contains 9969 triplets, spanning 6354 articles, for which the necessary EPA scores are available. This means that I can map the affective meanings onto these triplets, which in turn allows me to calculate their distance to the moral template.

To operationalize the notion of "negative moral vocabulary," I use the Moral Foundations Dictionary. This dictionary was compiled by Graham et. al. (2009) and provides a list of words for that are meant to capture the positive and negative aspects of each foundation, as well as a section of "general morality" terms. For this analysis, I use a list consisting only of the negative words; a total of 165 terms. The are two main reasons behind my decision to use this dictionary. First, even though this analysis is not situated within the framework of MFT, this dictionary is the result of the conscious effort of researchers who have done rigorous work on moral judgments. Therefore, it is a more than adequate starting point to examine "moral vocabulary." Second, the Moral Foundations Dictionary is freely available and easy to access. This renders my analysis reproducible and transparent.

My hypothesis posits a negative relationship between how distant an event is from the moral template and the amount of negative moral vocabulary that occurs around it. In order to build the dependent variable, I extract excerpts of text around each event and calculate the number of words that coincide with the negative terms from the Moral Foundations Dictionary. The excerpts consist of the fifty words prior to the first term of the triplet and the fifty words following it. Given that some triplets occur at the beginning or end of articles, the length of the resulting excerpt might vary. The length of the window here represents a trade-off. A narrow window might not be enough to capture all of the moral vocabulary used around the events, but casting a wide net might result in too many false positives - i.e. negative moral terms that might be related to other triplets. I conducted the same analysis below using windows of 50, 25 and 10 words (available in the supplemental materials) without appreciable differences in the results. I settle on 50 words for the analysis 
here because it provides a good balance between relative proximity around the triplet and enough width to capture moral vocabulary.

Before checking for matches, I lemmatize and stem the words in both the excerpts and the dictionary. This helps identify overlaps, even if modified versions or alternative spellings of the words are used. In this analysis, the outcome variable represents a count and, therefore, a Poisson regression is the most appropriate. Thus, I regress the count of matches on the event's distance from the template, controlling for the length of the excerpt and the overall sentiment of the text, which I calculate using the NLTK library's sentiment analyzer (VADER). I also include varying intercepts for each article.

\section{Results}

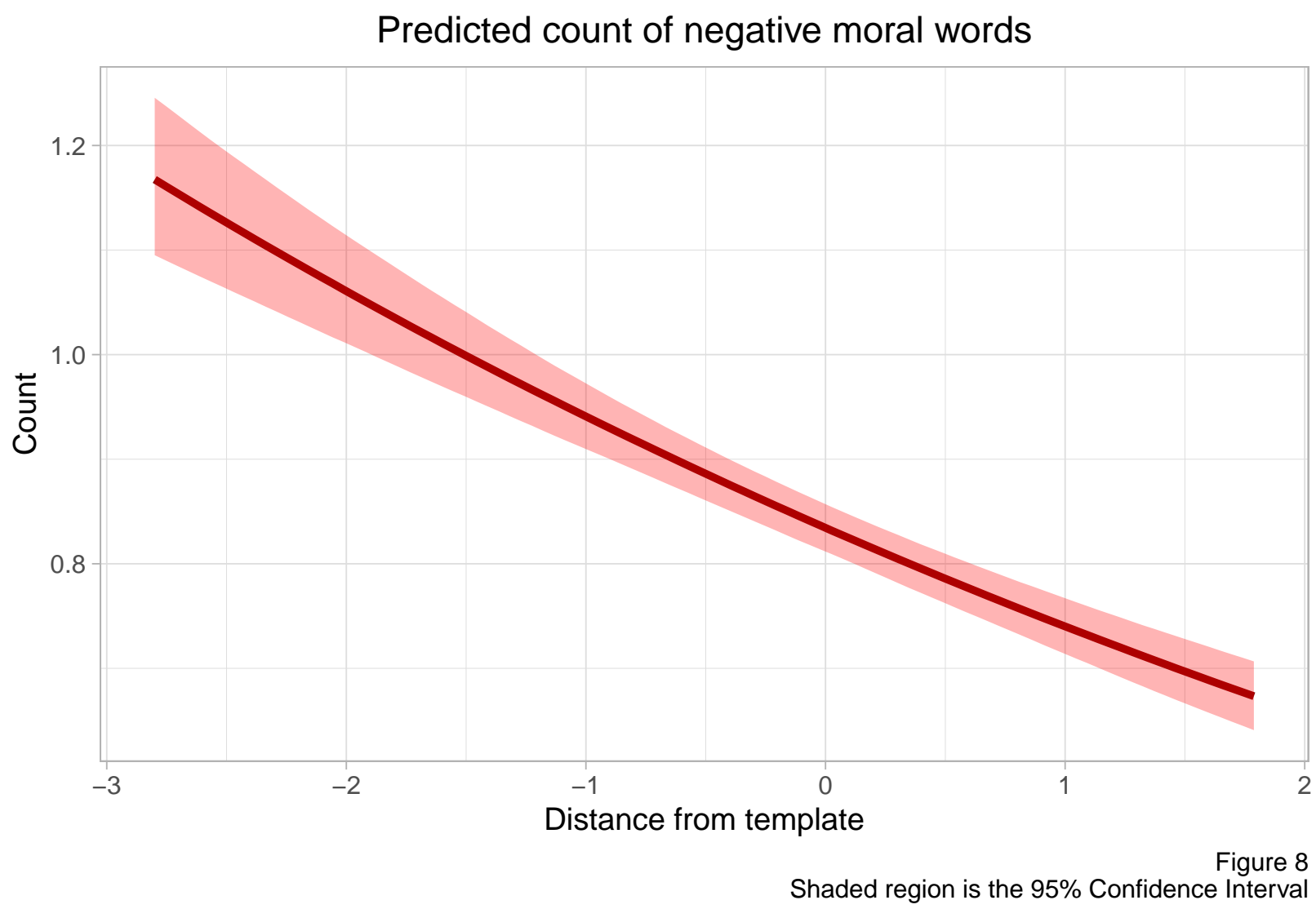

Figure 8 shows the predicted count of negative moral words when holding length of the excerpt and overall sentiment at their medians. As hypothesized, there is a negative relationship. An 
event that is two standard deviations closer than average to the template has a predicted count of about 1.2 negative moral words. In contrast, a triplet that is two standard deviations further away than average is predicted to have around 0.6 negative moral terms surrounding it. Although the effect size is relatively small, this finding lends credence to the idea that distance from the moral template is useful for understanding the moral language that cooccurs around an event. This is especially apparent when we consider that distance from the template remains informative even when we have taken general sentiment into account. The connotative structures of the events are not only predictive of whether an event is presented in a generally positive or negative light. Rather they give us additional information about the moral language that is being used around the triplets. These findings, then, give plausibility to the hypothesis outlined above, thus also affirming the external validity of the results of Studies 1 and 2 .

\section{Discussion}

This project uses tools from cultural sociology to adjudicate a central debate that exists in interdisciplinary work about moral cognition. The debate is, to a great extent, about categorization: whether moral judgments are a result of contrasting the events we witness with a mental template of the typical transgression. One of the main obstacles when it comes to testing this theory is outlining a principled definition of a typical moral transgression. But realizing that mental templates are cultural - and therefore shared - turns this problem into an issue of measuring meaning; a well-trodden territory for cultural sociologists (Edelmann 2018). The majority of work on moral cognition has used hypothetical scenarios to elicit moral judgments from participants (Clifford 2017). Here, I show that we can use the dictionaries of semantic profiles collected by ACT scholars to translate vignettes into formats that allow us to explore their connotative structures. Doing so yields insightful findings. For instance, Study 1 shows that, echoing Gray and Schein's (2018) arguments, transgressions have shared regularities: they involve a negative and potent behavior directed towards a weak object. We 
can bring rigor to their definition of an immoral template: a damaging behavior could be considered an action which is low on evaluation but high on potency; a vulnerable patient could be described as an actor who has a low potency score.

By establishing connotative regularities of moral transgressions, I outline a formal definition of what the template of an immoral act looks like and calculate deviations from it. Then I test the proposition that moral cognition occurs through template matching. The results from Study 2 show that distance from the template is informative of how long it takes individuals to classify events as harmful. Interestingly, the results from Study 2 also suggest that the relationship is quadratic: as distance increases, reaction time also increases, but only up to a point. Individuals are quick to classify events that are very close or far apart from the template. Events that lie at a middling distance are more ambiguous and take longer to be classified. These findings provide empirical evidence for the notion that template matching mediates moral cognition, and also give a clearer idea of how this process occurs.

Study 3 shows that these ideas are informative when considering a wider set of events, extracted from naturally occurring text. Using tools from computational natural language processing, it is possible to extract subject-verb-object triplets from text corpora. These triplets, in turn, have the same grammatical structure as the template I constructed, which allows for their "translation." The results from Study 3 provide evidence for the external validity of the survey findings. The ability to map connotative meanings to naturally occurring text opens up the possibility of testing a host of hypotheses about morality. Here, I show that by looking at the connotative structures of triplets, it is possible to gain knowledge about the negative moral vocabulary that occurs around them. The possibilities increase further when we consider that there is an active strand of research that attempts - quite successfully - to use machine learning techniques to derive EPA scores for terms that are not in the collected dictionaries (Loon and Freese 2019). This would allow us to explore the connotative structures underpinning moral judgments in a much wider range of settings. 
These studies also shed light on another important issue within the study of moral cognition. Many of the theoretical developments in the study of morality have been animated by the question of whether there are such things as harmless wrongs (Tepe and Aydinli-Karakulak 2019). The results from Study 1 side with Gray and Schein (2018), noting that some of the so-called "purity" transgressions are perceived as harmful. Yet, the findings from Study 2 compel us to rethink the distinctiveness of these scenarios. These violations do not match the patterns that the models uncover in the data; they are distant from the template and very harmful. Both sides of the debate, then, have pointed out important aspects of this type of transgressions. Gray and Schein (2018) are right in insisting that that purity scenarios are understood through the lens of harm. However, these transgressions are distinctive insofar as their semantic structures do not match their perceived harmfulness. My findings suggest that we should move away from the debate about "harmless" wrongs, but they compel us to consider how practices that are distant from the moral template come to be regarded as harmful (Schein and Gray 2016).

At a more general level, this work illustrates that sociological approaches to culture and cognition have the potential to meaningfully contribute to interdisciplinary debates surrounding categorization. For the last three decades, sociologists have imported ideas from cognitive science and psychology to advance our understanding of how beliefs and actions are shaped by the cultural contexts where individuals find themselves. This dialogue has been productive but insights and tools that have resulted from it have largely remained within disciplinary boundaries. As Vaisey and Valentino (2018) have noted, despite considerable common ground, sociologists seldom venture to contribute to more interdisciplinary efforts to understand judgments and action. Here, I show that sociological work about how to conceptualize and measure cultural categories can be exported and used to test key research questions. Testing the role of template matching in moral decision-making becomes a tractable task when mental templates are understood as cultural objects, which are widely shared and, therefore, measurable. Using tools from cultural sociology, it is possible to address one of the central 
debates in the interdisciplinary study of morality. This paper, then, shows that it would be fruitful for cultural sociologists to look outward: to dialogue with other interdisciplinary research agendas, where their tools and ideas might help answer some of the most pressing questions.

\section{Limitations}

An important limitation of Studies 1 and 2 concerns the number and type of fictional vignettes used. Given that I only use 25 vignettes, I rely on limited variation to produce my estimates. Furthermore, the events are all practices that are relatively immoral. Effectively what I am doing is a form of selecting on the dependent variable. Study 3 tries to ameliorate this by testing the findings on a wider set of events, showing the external validity of the results. However, I arrived at the hypothesis using a limited sample of events. Future work should focus on exploring events with widely varying connotative structures.

Another potential shortcoming of this analysis is related to the sensitivity of one of the instruments. Reaction time studies are fickle and here I am adding complexity to this task. I am asking participants to react to full sentences and to do so remotely. Though the data show consistent patterns, these limitations should be taken seriously. In future work, it would be useful to find ways of measuring moral cognition more precisely.

Lastly, Study 3 shows the possibilities that natural language processing offers to the study of morality but these techniques are prone to error. For instance, the parts-of-speech tools I implement to extract the triplets cannot recognize sentences with complex structures. Moreover, tools like sentiment analysis are correctly identified as blunt, incapable of discerning important context cues like irony. Despite these shortcomings, the results provide important insights, showing that researchers can use naturally occurring text to probe at interesting questions in the study of morality. 


\section{Conclusion}

These studies show that a sociological approach to measuring meaning helps us provide a concrete test of one of the most plausible contemporary theories of moral cognition. Current evidence supports a more unified vision of moral judgment, where perceptions of harm are the main drivers of this process. Gray and Schein have outlined a model of moral cognition that is consistent with this vision, where immorality is ascribed through template matching. The evidence to support this position, however, remains limited. Here, I show that we can use tools from cultural sociology to test it. I use existent repositories of cultural meanings to examine the connotative structures of moral transgressions. This allows me to accomplish two things: to produce a rigorous definition of a prototypical moral wrong, and to examine whether deviations from it affect how individuals perceive transgressions. The results suggest that distance from the prototypical moral transgression is informative of the time it takes individuals to classify scenarios as harmful. Furthermore, distance from the template is also predictive of the negative moral vocabulary around events in naturally occurring text. This paper illustrates that sociological discussions about cultural categories - and how to go about measuring them - have the potential to contribute to key interdisciplinary debates.

\section{References:}

Bergstrand, Kelly. 2019. "The Advantaged Cause: Affect Control Theory and Social Movements." Socius: Sociological Research for a Dynamic World 5 (January): 237802311984181. https://doi.org/10.1177/2378023119841811.

Brubaker, Rogers, Mara Loveman, and Peter Stamatov. 2004. "Ethnicity as Cognition." Theory and Society 33 (1): 31-64. https://doi.org/10.1023/B:RYSO.0000021405.18890.63.

Clifford, Scott. 2017. "Individual Differences in Group Loyalty Predict Partisan Strength." Political Behavior 39 (3): 531-52. https://doi.org/10.1007/s11109-016-9367-3. 
Clifford, Scott, Vijeth Iyengar, Roberto Cabeza, and Walter Sinnott-Armstrong. 2015. "Moral Foundations Vignettes: A Standardized Stimulus Database of Scenarios Based on Moral Foundations Theory." Behavior Research Methods 47 (4): 1178-98.

Edelmann, Achim. 2018. "Formalizing Symbolic Boundaries.” Poetics 68: 120-30.

Graham, Jesse, Jonathan Haidt, Sena Koleva, Matt Motyl, Ravi Iyer, Sean P. Wojcik, and Peter H. Ditto. 2013. "Moral Foundations Theory: The Pragmatic Validity of Moral Pluralism." In Advances in Experimental Social Psychology, 47:55-130. Elsevier.

Graham, Jesse, Jonathan Haidt, and Brian A. Nosek. 2009. "Liberals and Conservatives Rely on Different Sets of Moral Foundations." Journal of Personality and Social Psychology 96 (5): 1029 .

Gray, Kurt, and Jonathan E. Keeney. 2015a. "Impure or Just Weird? Scenario Sampling Bias Raises Questions about the Foundation of Morality." Social Psychological and Personality Science 6 (8): 859-68.

—. 2015b. "Disconfirming Moral Foundations Theory on Its Own Terms: Reply to Graham (2015)." Social Psychological and Personality Science 6 (8): 874-77. https: //doi.org/10.1177/1948550615592243.

Gray, Kurt, Liane Young, and Adam Waytz. 2012. "Mind Perception Is the Essence of Morality." Psychological Inquiry 23 (2): 101-24. https://doi.org/10.1080/1047840X.2012. 651387.

Greene, Joshua D. 2014. "Beyond Point-and-Shoot Morality: Why Cognitive (Neuro)Science Matters for Ethics." Ethics 124 (4): 695-726. https://doi.org/10.1086/675875.

—. 2015. "The Rise of Moral Cognition." Cognition 135 (February): 39-42. https: //doi.org/10.1016/j.cognition.2014.11.018. 
Haidt, Jonathan, and Craig Joseph. 2004. "Intuitive Ethics: How Innately Prepared Intuitions Generate Culturally Variable Virtues." Daedalus 133 (4): 55-66. https: //doi.org/10.1162/0011526042365555.

Haidt, Jonathan, Silvia Helena Koller, and Maria G. Dias. 1993. "Affect, Culture, and Morality, or Is It Wrong to Eat Your Dog?" Journal of Personality and Social Psychology 65 (4): 613-28. https://doi.org/10.1037/0022-3514.65.4.613.

Harnad, Stevan. 2017. "To Cognize Is to Categorize: Cognition Is Categorization." In Handbook of Categorization in Cognitive Science, 21-54. Elsevier.

Heise, David R. 2007. Expressive Order: Confirming Sentiments in Social Actions. Springer Science \& Business Media.

Heise, David R. 2014. "Cultural Variations in Sentiments." SpringerPlus 3 (1). https: //doi.org/10.1186/2193-1801-3-170.

Hunzaker, M. B. Fallin. 2016. "Cultural Sentiments and Schema-Consistency Bias in Information Transmission." American Sociological Review 81 (6): 1223-50. https: //doi.org/10.1177/0003122416671742.

Hunzaker, MB Fallin, and Lauren Valentino. 2019. "Mapping Cultural Schemas: From Theory to Method." American Sociological Review 84 (5): 950-81.

Joseph, Kenneth, Wei Wei, Matthew Benigni, and Kathleen M. Carley. 2016. "A Social-Event Based Approach to Sentiment Analysis of Identities and Behaviors in Text." The Journal of Mathematical Sociology 40 (3): 137-66.

Lamont, Michèle. 1992. Money, Morals, and Manners: The Culture of the French and the American Upper-Middle Class. University of Chicago Press.

Loon, Austin van, and Jeremy Freese. 2019. "Can We Distill Fundamental Sentiments from Natural Language Use? Evaluating Word Embeddings as a Complement to Survey-Based 
Ratings of Affective Meaning." July. https://doi.org/10.31235/osf.io/r7ewx.

Martin, John Levi, and Matthew Desmond. 2010. "Political Position and Social Knowledge." Sociological Forum 25 (1): 1-26. https://doi.org/10.1111/j.1573-7861.2009.01154.x.

Monk, Ellis P. 2019. "The Color of Punishment: African Americans, Skin Tone, and the Criminal Justice System." Ethnic and Racial Studies 42 (10): 1593-1612.

Moore, Rick. 2017. "Fast or Slow: Sociological Implications of Measuring Dual-Process Cognition." Sociological Science 4: 196-223. https://doi.org/10.15195/v4.a9.

Osgood, Charles E. 1969. "On the Whys and Wherefores of E, P, and A." Journal of Personality and Social Psychology 12 (3): 194-99. https://doi.org/10.1037/h0027715.

Osgood, Charles Egerton, William H. May, Murray Samuel Miron, and Murray S. Miron. 1975. Cross-Cultural Universals of Affective Meaning. Vol. 1. University of Illinois Press.

Peer, Eyal, Laura Brandimarte, Sonam Samat, and Alessandro Acquisti. 2017. "Beyond the Turk: Alternative Platforms for Crowdsourcing Behavioral Research." Journal of Experimental Social Psychology 70 (May): 153-63. https://doi.org/10.1016/j.jesp.2017.01 .006 .

Robinson, Dawn T, Lynn Smith-Lovin, Bryan Cannon, Jesse K. Clark, Robert E. Freeland, Jonathan H. Morgan, and Kimberly B. Rogers. 2016. "Mean Affective Ratings of 929 Identities, 814 Behaviors, and 660 Modifiers by University of Georgia and Duke University Undergraduates and by Community Members in Durham, NC, in 2012-2014." Distributed at UGA Affect Control Theory Website.

Rosch, Eleanor. 1988. "Principles of Categorization." In Readings in Cognitive Science, 312-22. Elsevier. https://doi.org/10.1016/B978-1-4832-1446-7.50028-5.

Schein, Chelsea, and Kurt Gray. 2016. "Moralization and Harmification: The Dyadic Loop Explains How the Innocuous Becomes Harmful and Wrong." Psychological Inquiry 27 (1): 
$62-65$.

— . 2018. "The Theory of Dyadic Morality: Reinventing Moral Judgment by Redefining Harm." Personality and Social Psychology Review 22 (1): 32-70. https://doi.org/10.117 $7 / 1088868317698288$.

Schröder, Tobias, Kimberly B. Rogers, Shuichirou Ike, Julija N. Mell, and Wolfgang Scholl. 2013. "Affective Meanings of Stereotyped Social Groups in Cross-Cultural Comparison." Group Processes 8 Intergroup Relations 16 (6): 717-33. https://doi.org/10.1177/136843 0213491788 .

Tepe, Beyza, and Arzu Aydinli-Karakulak. 2019. "Beyond Harmfulness and Impurity: Moral Wrongness as a Violation of Relational Motivations." Journal of Personality and Social Psychology 117 (2): 310-37. https://doi.org/10.1037/pspi0000169.

Vaisey, Stephen, and Lauren Valentino. 2018. "Culture and Choice: Toward Integrating Cultural Sociology with the Judgment and Decision-Making Sciences." Poetics 68: 131-43.

Young, Liane, and Rebecca Saxe. 2011. "When Ignorance Is No Excuse: Different Roles for Intent Across Moral Domains." Cognition 120 (2): 202-14. https://doi.org/10.1016/j.co gnition.2011.04.005.

Zerubavel, Eviatar. 1996. "Lumping and Splitting: Notes on Social Classification." In Sociological Forum, 11:421-33. Springer. 